\title{
Identificación de especies de Malassezia aisladas de piel sana en pobladores de Lima, Perú
}

\author{
Identification of Malassezia species isolated from healthy skin in residents of Lima, Peru
}

\author{
Vilma Bejar ${ }^{1,2}$, Carlos Rojas ${ }^{2}$, Jose M. Guevara G. ${ }^{1,2,3}$, Elizabeth Pareja ${ }^{1,2}$, \\ Ana Huaman ${ }^{1,2}$, Raul Sevilla ${ }^{2}$, Mario Tapia ${ }^{2}$, Rito Zerpa ${ }^{1,2}$, Sofía Gonzalez ${ }^{1,2,4}$, \\ Freddy Villanueva ${ }^{2,6}$, Esther Valencia ${ }^{1}$, Luis Marocho ${ }^{2,4}$ y Enma Abanto ${ }^{1}$ \\ 'Instituto de Medicina Tropical 'Daniel A. Carrión', Facultad de Medicina, Universidad Nacional Mayor de San Marcos, Lima, Perú. \\ ${ }^{2}$ Departamento de Microbiología Médica, Facultad de Medicina, UNMSM, Lima, Perú. \\ ${ }^{3}$ Hospital Daniel Alcides Carrión, Lima, Perú. \\ ${ }^{4}$ Centro Médico Naval 'Cirujano Mayor Santiago Távara', Lima, Perú. \\ ${ }^{5}$ Instituto Nacional de Salud del Niño, Lima, Perú. \\ ${ }^{6}$ Instituto Nacional de Enfermedades Neoplásicas, Lima, Perú.
}

\begin{abstract}
Resumen
Objetivo: Identificar las especies de Malassezia en zonas seborreicas de piel sana en población limeña. Diseño: Estudio descriptivo transversal. Lugar: Instituto de Medicina Tropical 'Daniel Alcides Carrión', Universidad Nacional Mayor de San Marcos, Lima, Perú. Participantes: Pobladores asintomáticos. Intervenciones: Empleando la técnica de Mariat y Adan-Campos, se recolectó muestras de piel de 129 pobladores asintomáticos de diversos distritos de la ciudad de Lima. El aislamiento de Malassezia se realizó en medio Dixon modificado e incubado a $32^{\circ} \mathrm{C}$ por 7 dias, la identificación de las colonias por sus características macro y micromorfológicas, y la tipificación mediante el estudio de las propiedades bioquímicas y fisiológicas según la técnica de Guillot y col. Principales medidas de resultados: Especie de Malassezia, sexo, edad y región anatómica. Resultados: Se aisló Malassezia spp en 43,4\% de los pobladores, obteniéndose $49,2 \%$ en varones y $37,5 \%$ en mujeres. De las diferentes regiones corporales, 68 cultivos fueron positivos: cuero cabelludo $31(45,6 \%)$, espalda $36(52,9 \%)$ y región frontal $1(1,5 \%)$. El grupo etario con mayor frecuencia de aislamientos $(47,2 \%)$ fue el de 14 a 25 años (adolescentes jóvenes). M. slooffiae fue encontrado en $83,8 \%$ y M. obtusa en $16,2 \%$ de los casos. Conclusiones: Se encontró Malassezia spp. en la piel humana sana. M. slooffiae fue la especie predominante de los casos positivos $(83,8 \%)$ seguido de M. obtusa (16,2\%).
\end{abstract}

Palabra clave: Malassezia spp, piel sana, asimilación de tween.

\section{Abstract}

Objective: To identify Malassezia species in healthy skin seborrhea areas in Lima inhabitants. Design: Cross-sectional study. Setting: Daniel Alcides Carrion Tropical Medicine Institute, Universidad Nacional Mayor de San Marcos, Lima, Peru. Participants: Asymptomatic persons. Interventions: Skin samples were collected from 129 asymptomatic residents of several districts of Lima city using Mariat and Adan-Campos' technique. Malassezia isolation was performed in modified Dixon medium and incubated at $32^{\circ} \mathrm{C}$ for 7 days. Colonies were identified by macro and micro morphological characteristics and typing was determined by biochemical and physiological properties using Guillot's technique. Main outcome measures: Malassezia species, participants' gender, age and anatomical region. Results: Malassezia spp was isolated in $43.4 \%$ of the residents, $49.2 \%$ in men and $37.5 \%$ in women. From various body regions 68 cultures were positive: scalp 31 (45.6\%), back $36(52.9 \%)$ and frontal region $1(1.5 \%)$. Isolates most common age group (47.2\%) was that of adolescents-young (14-25 year-old). M. slooffiae was found in $83.8 \%$ and M. obtusa in $16.2 \%$ of cases. Conclusions: Malassezia spp. was present in healthy human skin. M. slooffiae was the predominant species in positive cases (83.8\%) followed by M. obtusa (16.2\%).

Keywords: Malassezia spp, healthy skin, Tween assimilation.

An Fac med. 2014;75(2):173-6 / doi: http://dx.doi.org/10.15381/anales.v75i2.8347 


\section{INTRODUCCIÓN}

El género Malassezia incluye levaduras que forman parte de la flora normal de la piel humana y de otros animales de sangre caliente, siendo lipófilas, ya que para su desarrollo requieren de ácidos grasos de cadena media y larga como fuente de carbono, exceptuándose a $\mathrm{M}$. pachydermatis que es la única especie no lípido-dependiente ${ }^{(1)}$.

La fase micelial de estas levaduras fue designada por muchos años con el nombre de Malassezia furfur, mientras que a los dos tipos morfológicos de la fase de levadura se les llamó Pityrosporum ovale y $P$. orbiculare. Con el desarrollo de las técnicas moleculares y estudios morfológicos, bioquímicos y fisiológicos se sabe que el taxón Malassezia comprende 14 especies: 7 de ellas descritas por Guého y col. (2): (Malassezia furfur, M. globosa, M. obtusa, M. restricta, $M$. slooffiae, $M$. sympodialis y M. pachydermatis), 4 descubiertas en investigaciones japonesas (Malassezia dermatis ${ }^{(3)}$, M. yamatoensis ${ }^{(4)}$, M. japonica ${ }^{(5)}$ y M. nana ${ }^{(6)}$ ) y 3 nuevas especies aisladas de animales (M. caprae, M. equine ${ }^{(7)}$ y cuniculi $\left.{ }^{(8)}\right)$.

En las últimas décadas, Malassezia spp. se ha visto implicada, además de la pitiriasis versicolor, como agente causal de otros cuadros clínicos, como la dermatitis seborreica, dermatitis atópica, foliculitis y fungemia en pacientes con catéteres intravasculares ${ }^{(9,10)}$. Su incidencia es 30 a $50 \%$ en países con clima tropical, mientras en climas templados oscila entre $1,1 \%$ y $3,7 \%$, representando alrededor del $20 \%$ de las micosis superficiales y siendo más frecuente en verano y otoño ${ }^{(11)}$.

Además de sus características morfológicas, las especies del género Malassezia se diferencian por su capacidad de asimilar diferentes esteres presentes en Tween 20, 40, 60 y 80, según la metodología de Guillot y col. (12), lo que se explica según estudios de $\mathrm{Xu}$ y col ${ }^{(13)}$ del genoma y del proteoma secretor; estos autores sugirieron que la lípido dependencia ocurre por la falta de genes que sinteticen ácidos grasos. La identi- ficación de estas especies y su prevalencia ha sido estudiada en diversos países cuyas condiciones socio-económicas, además de su clima propio, son diferentes al nuestro. Por lo anterior, se realizó este estudio, con el objetivo de conocer la frecuencia de las especies de Malassezia en zonas seborreicas de piel sana.

\section{MÉTODOS}

Se realizó un estudio descriptivo transversal en 129 personas asintomáticas, de los diferentes distritos de la ciudad de Lima, de quienes se obtuvo el consentimiento informado y/o autorización de los padres o tutores, durante el periodo de abril y julio de 2011. La muestra comprendió 65 varones y 64 mujeres, cuyas edades oscilaron entre los 2 y 78 años; se les agrupó según las etapas del desarrollo humano: a) grupo 1, hasta el final de la pubertad (de 2 a 13 años, $\left.\mathrm{n}_{1}=26\right)$; b) grupo 2, adolescencia-juventud (de 14 a 25 años, $n_{2}=53$ ); y, c) grupo 3, adultez-senectud (de 26 a 78 años, $\mathrm{n}_{3}=50$ ).

Las muestras fueron recolectadas usando la técnica de Mariat y AdanCampos ${ }^{(14)}$, que consiste en frotar con un tapiz estéril la piel sana de las regiones seborreicas, es decir, cuero cabelludo, región frontal, tronco y espalda. Las

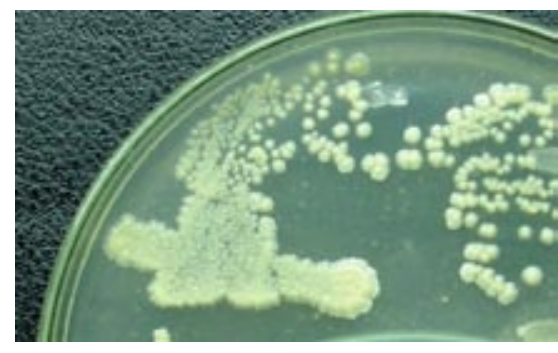

Figura 1. Crecimiento de Malassezia spp en medio Dixon modificado.

muestras fueron cultivadas en placas de agar sabouraud dextrosa (ASD) suplementado con aceite de oliva - bilis de buey e incubadas a $32^{\circ} \mathrm{C}$, y en ASD con cloramfenicol, incubándose a temperatura ambiental por una semana; de este modo se diferenció el crecimiento de las levaduras lipofílicas.

Los cultivos positivos a Malassezia spp. fueron confirmados por el examen directo de las colonias levaduriformes.

La identificación de las especies se realizó mediante el estudio de la morfología macroscópica y microscópica, y las propiedades fisiológicas y bioquímicas, según los criterios de Guého y col (2) y Guillot y col ${ }^{(12)}$. Para ello se realizó previamente la resiembra de las colonias a una suspensión de $10^{5}$ levaduras $/ \mathrm{mL}$ en agua destilada estéril en medio Dixon modificado (peptona 0,6\%; extracto de

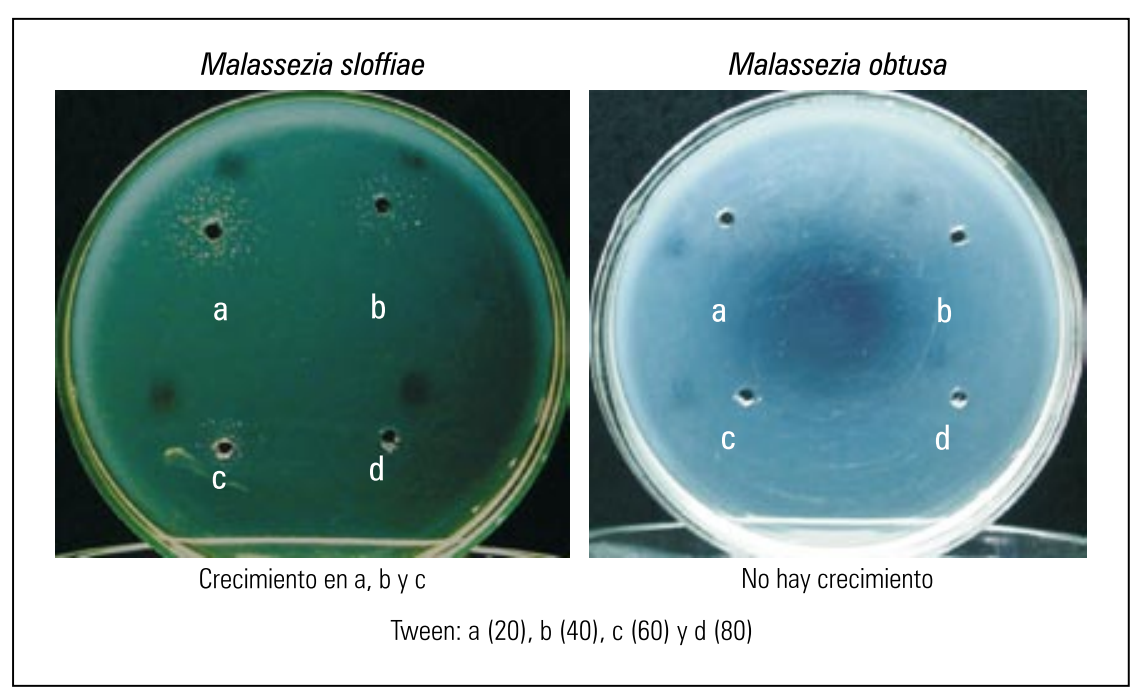

Figura 2. Pruebas de asimilación de Tween. 
malta 3,6\%; bilis de buey 2,0\%; Tween 40 1,0\%; ácido oleico 0,2\%; glicerol $0,2 \%$; agar $1,2 \%$; pH 6,0$)$, a $32^{\circ} \mathrm{C}$, por siete días, con el fin de detectar posible diversidad en la morfología de las colonias (figura 1); y el estudio de las características microscópicas se hizo mediante coloración Gram.

A partir de cultivos de tres días en agar mDixon se realizó pruebas bioquímicas y fisiológicas, como la reacción de la catalasa; el crecimiento en ASD simple a $32^{\circ} \mathrm{C}$ y en agar mDixon a diferentes temperaturas $\left(32,37\right.$ y $\left.40^{\circ} \mathrm{C}\right)$. Para la prueba de asimilación de Tween 20, 40, 60 y 80 se mezcló $16 \mathrm{~mL}$ de ASD estéril, a una temperatura menor a $50^{\circ} \mathrm{C}$, con $2 \mathrm{~mL}$ de una suspensión de las levaduras equivalente a $10^{5}$ células/ $\mathrm{mL}$, en agua destilada. El agar fue luego mezclado y depositado en una placa petri estéril de $9 \mathrm{~cm}$ de diámetro. Cuando solidificó el medio, se hizo 4 pocillos de $2 \mathrm{~mm}$ de diámetro, en los que se adicionó $5 \mu \mathrm{L}$ de Tween de 20, 40, 60 y 80, respectivamente, incubándose a $32^{\circ} \mathrm{C}$ y realizando la lectura a los 7 días (figura 2). Un resultado fue considerado positivo cuando se observó el desarrollo de las colonias alrededor de los pocillos con el Tween respectivo.

\section{RESULTADOS}

De los raspados de piel sana de 129 pobladores, $56(43,4 \%)$ tuvieron cultivos positivos a Malassezia spp., obteniéndose 68 aislamientos de diferentes regiones corporales. Del raspado de cuero cabelludo se obtuvo 31 (45,6\%), de espalda $36(52,9 \%)$ y de región frontal $1(1,5 \%)$ aislamientos, no existiendo aislamiento en tronco. En 12 pobladores se aisló Malassezia spp. en 2 regiones corporales.

De 65 varones, 32 (49,2\%) tuvieron cultivo positivo; del mismo modo, en $24(37,5 \%)$ de las 64 mujeres incluidas en el estudio se aisló Malassezia spp.

Respecto a la edad, se encontró que el grupo 1 presentó $38,5 \%$, el grupo 2 , $47,2 \%$, y el grupo $3,42 \%$ cultivos positivos.

Tabla 1. Caracterización bioquímica y fisiológica de las Malassezia aisladas de piel sana.

\begin{tabular}{|c|c|c|c|c|c|c|c|c|c|}
\hline \multirow{2}{*}{ Especies } & \multirow{2}{*}{ Catalasa } & \multirow{2}{*}{$\begin{array}{l}\text { ASG* }^{*} \\
320 \mathrm{C}\end{array}$} & \multicolumn{3}{|c|}{ Agar mDixon } & \multicolumn{4}{|c|}{ Asimilación Twin } \\
\hline & & & $320 \mathrm{C}$ & $370 C$ & $400 C$ & 20 & 40 & 60 & 80 \\
\hline Malassezia obtusa & + & - & + & + & - & - & - & - & - \\
\hline Malassezia slooffiae & + & - & + & + & + & + & + & + & - \\
\hline
\end{tabular}

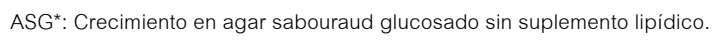
mDixon: Crecimiento en agar Dixon modificado.

Tabla 2. Distribución porcentual de las especies de Malassezia según región corporal.

\begin{tabular}{cccccc} 
& \multicolumn{2}{c}{ Cuero cabelludo $(\mathrm{n}=31)$} & & \multicolumn{2}{c}{ Espalda $(\mathrm{n}=36)$} \\
\cline { 2 - 3 } \cline { 5 - 6 } Especie & $\mathrm{n}$ & $\%$ & & $\mathrm{n}$ & $\%$ \\
M. slooffiae & 25 & 80,6 & & 31 & 86,1 \\
M. obtusa & 6 & 19,4 & & 5 & 13,9 \\
\hline
\end{tabular}

De los cultivos positivos a Malassezia, se identificó dos especies: M. Obtusa y M slooffiae (tabla 1).

La tipificación de especies mostró a Malassezia slooffiae en 57 aislamientos $(83,8 \%)$ y a Malassezia obtusa en 11 $(16,2 \%)$ del total de cultivos positivos (figura 3). Según la zona corporal, tanto en cuero cabelludo como espalda la mayor frecuencia fue de Malassezia slooffiae (tabla 2) y el único aislamiento en región frontal también correspondió a esta especie.

\section{DISCUSIÓN}

Durante muchos años Malassezia spp. ha sido considerada una levadura de patogenia y distribución desconocida, debido a la dificultad que hubo en un inicio de cultivarla, además de la falta de un método que permitiera la identificación de las diferentes especies de este género ${ }^{(9)}$. Luego de los estudios de Guého y col ${ }^{(2)}$ y Guillot y col ${ }^{(12)}$, aquello fue posible. Sin embargo, las publicaciones con respecto a la prevalencia de las diferentes especies, en piel sana o con diversas dermatosis que ocasiona, es aún escasa, relacionándose solo a M. furfur en las diferentes condiciones mórbidas $(7,11,15,16)$.

Nuestros hallazgos positivos para Malassezia spp en $43,4 \%$ de nuestra muestra comparados con el 69,4\% obtenido por Crespo y col. en un estudio realizado en Europa (España), deben ser analizados considerando la realidad socio-económica y climática de cada región. Por otro lado, a pesar de considerarse a Lima como una ciudad de clima templado, el porcentaje hallado se encuentra muy por encima de los porcentajes esperados $(1,1 \%$ a $3,7 \%)$, más aun cuando las muestras fueron colectadas en invierno y primavera.

Así mismo, se observa un mayor porcentaje de aislamientos positivos en los varones $(49,2 \%)$ en comparación a las mujeres $(37,5 \%)$ explicándose esto por las características propias de la piel masculina donde las áreas seborreicas son de mayor actividad por motivos hormonales. Esto último también explica el ligero predominio de aislamientos positivos en el grupo 2, que comprendía a los pobladores de 14 a 25 años, es decir, los adolescentes y jóvenes, en quienes debido a su desarrollo físico y los cambios hormonales provocan un aumento de la actividad de las glándulas sebáceas, beneficiando en ellos la presencia de Malassezia spp. como parte de su flora normal. No podemos dejar de mencionar, que reagrupando nuestros datos y considerando solo la etapa púber (de 9 a 13 años), donde se inician estos cambios y con mayor intensidad, los aislamientos positivos llegan a 50\%. 
Nuestro estudio muestra un predominio de M. slooffiae, en $83,8 \%$, seguido de M. obtusa con 16,2\% de los 68 aislamientos, a diferencia de los estudios de Hernández y col (16) en una población mexicana, donde es M. restricta $(47,6 \%)$ la especie predominante, por encima de M. globosa $(23,8 \%)$ y de M. sympodialis y M. slooffiae, en menor porcentaje. Del mismo modo, Crespo y col ${ }^{(9)}$, en España, informan un predominio de M. sympodialis en $88,5 \%$ y M. globosa en $11,5 \%$ de raspados de piel sana.

En este último estudio además se informa que en la región escapular solo se aisló M. sympodialis (100\%) en 21 cultivos positivos de un total de $25 \mathrm{pa}$ cientes, a diferencia nuestra en la que aislamos M. slooffiae $(86,1 \%)$ y M. obtusa $(13,9 \%)$ en 36 cultivos positivos de un total de 129 pacientes. Así mismo, obtuvimos un predominio de M. slooffiae $(80,6 \%, \mathrm{~N}=25)$ en cuero cabelludo y un solo aislamiento de esta en la región frontal.

En conclusión, en la población de Lima los resultados demuestran la presencia de Malassezia spp. en piel sana, siendo M. slooffiae la especie más frecuente seguida de M. obtusa, teniendo ambas como hábitat preferente la piel humana. Aunque, también se sabe que M. slooffiae junto a M. furfur se relacionan a la piel de animales, lo que implica un mayor contacto con animales que muchas familias poseen en el hogar, siendo necesario un estudio que demuestre la posible relación existente en estos casos, y el mayor predominio de
M. slooffiae en nuestra población sana. Así mismo, se recomienda realizar la tipificación molecular para confirmar la identificación de las especies encontradas, ya que posee un mayor poder de discriminación y confiabilidad, más aún ante la carencia de estudios previos que hayan investigado la microbiota de Malassezia en personas asintomáticas de nuestra población.

\section{REFERENCIAS BIBLIOGRÁFICAS}

1. Kindo AJ, Sophia SKC, Kalyani J, Anandan S. Identification of Malassezia species. Indian J Med Microbiol. 2004;22 (3):179-81.

2. Guého E, Midgley G, Guillot J. The genus Malassezia with description of four new species. Antonie Leeuwenhoek. 1996;69:337-55. doi: http://dx.doi. org/10.1007/BF00399623

3. Sugita $T$, Takashima M, Shinoda $T$, Suto $T$, Unno $T$, Tsuboi R, Ogawa $H$, Nishikawa A. New yeast species, Malassezia dermatis, isolated from patients with atopic dermatitis. J Clin Microbiol. 2002;40:1363-7. doi: http://dx.doi.org/10.1128/ JCM.40.4.1363-1367.2002

4. Sugita T, Tajima M, Takashima M, Amaya M, Saito M, Tsuboi R, Nishikawa A. A new yeast, Malassezia yamatoensis, isolated from a patient with seborrheic dermatitis, and its distribution in patients and healthy subjects. Microbiol Immunol. 2004;48:579-83.doi: http://dx.doi.org/10.1111/ j.1348-0421.2004.tb03554.x

5. Sugita T, Takashima M, Kodama M, Tsuboi R, Nishikawa A. Description of a new yeast species, Malassezia japonica, and its detection in patients with atopic dermatitis and healthy subjects. J Clin Microbiol. 2003;41:4695-9. doi: http://dx.doi. org/10.1128/JCM.41.10.4695-4699.2003

6. Hirai A., Kano R, Makimura K, Duarte ER, Hamdan JS, Lachance MA, Yamaguchi H, Hasegawa A. Malassezia nana sp. nov., a novel lipid-dependent yeast species isolated from animals. Int $\mathrm{J}$ Syst Evol Microbiol. 2004;54:623-7. doi: http://dx.doi. org/10.1099/ijs.0.02776-0

7. Caba-es FJ, Theelen B, Castellá G, Boekhout T. Two new lipid-dependent Malassezia species from domestic animals. FEMS Yeast Res.
2007;7(6):1064-76. doi: http://dx.doi.org/10.1111/ j.1567-1364.2007.00217.x

8. Caba-es FJ, Vega S, Castellá G. Malassezia cuniculi sp. nov., a novel yeast species isolated from rabbit skin. Medical Mycol. 2011;49(1):40-8. doi: http://dx.doi.org/10.3109/13693786.2010.493562

9. Crespo V, Ojeda A, Vera A, Crespo A, Sánchez F. Aislamiento e identificación de Malassezia spp en pitiriasis versicolor, dermatitis seborreica y piel sana. Rev Iberoam Micol. 1999;16:S16-S21.

10. Curvale N, Botterel F, Legrand P, Guillot J, Bretagne S. Frequency of intravascular catheter colonization by Malassezia spp. in adult patients. Mycoses. 2004;47:491-4. doi: http://dx.doi. org/10.1111/j.1439-0507.2004.01047.x

11. Ballesté R, Fernández N, Calegari L, Gezuele E. Pitiriasis versicolor en lactantes. Rev Med Uruguay. 2000;16:257-60.

12. Guillot J, Gueho E, Lesourd M, Midgley G, Chevrier G, Dupont B. Identification of Malassezia species. A practical approach. J Mycol Med. 1996;6:103-10.

13. Xu J, Saunders CW, Hu P, Grant RA, Boekhout T, et al. Dandruff-associated Malassezia genomes reveal convergent and divergent virulence traits shared with plant and human fungal pathogens. PNAS. 2007;104:18730-5. doi: http://dx.doi. org/10.1073/pnas.0706756104

14. Mariat F, Adad-Campos C. La technique du carre de tapis, méthode simple de prélèvement dans les mycoses superficielles. Ann Inst Pasteur. 1967;113:666-8.

15. Aspiroz MC, Moreno LA, Rubio MC. Taxonomia de Malassezia furfur: estado de la cuestión. Rev Iberoam Micol. 1997;14:147-9.

16. Hernández F, Méndez LJ, Bazán E, Arévalo A, Valera A, López R. Especies de Malassezia asociadas a diversas dermatosis y a piel sana población mexicana. Rev Iberoam Micol. 2003;20:141-4.

Financiamiento: Recursos propios.

No existen conflictos de interés.

\section{Correspondencia:}

Vilma Ruth Béjar Castillo

Sección de Micología, Instituto de Medicina Tropical Daniel A. Carrión, Facultad de Medicina, UNMSM

José Santos Chocano No 199, Bellavista-Callao.

Teléfono: 619 7000-4410

Correo electrónico: vbejarc@unmsm.edu.pe 